\title{
Intraband Carrier Photoexcitation in Quantum Dot Lasers
}

\author{
P. Moreno, ${ }^{*}$ M. Richard, M. Rossetti, M. Portella-Oberli, L. H. Li, \\ B. Deveaud-Plédran, and A. Fiore ${ }^{\dagger}$ \\ Ecole Polytechnique Fédérale de Lausanne, Institute of Quantum Electronics and \\ Photonics, CH-1015 Lausanne, Switzerland
}

Received November 29, 2007; Revised Manuscript Received February 3, 2008

\begin{abstract}
We unveil the role of bound-to-continuum photoexcitation of carriers as a relevant process that affects the performance of quantum dot (QD) lasers. We present the response of an InAs/InGaAs QD laser to a sub-band gap pump, showing an unexpected depletion of the emitted photons. We relate this observation with carrier photoexcitation through additional transmission and photocurrent measurements. We provide a theoretical support to the experimental data and highlight the important role of this process in the laser characteristics.
\end{abstract}

The electronic structure of semiconductor quantum dots (QDs) presents dramatic differences from the electronic structure of the bulk material from which they are derived, exhibiting unique properties for their use in lasers and other photonic devices. Besides interband optical transitions commonly used for laser operation, intraband transitions can also occur in QDs and have been applied to QD infrared photodetectors. ${ }^{1-3}$ For photon energies in the near-infrared, these transitions involve final states high in the continuum and produce an optical loss (corresponding to free-carrier absorption in bulk materials) for the wave propagating in the laser cavity. While in bulk, intraband transitions are forbidden in the first order as they do not conserve momentum, in QDs, the localized nature of the initial bound state makes the transition allowed for any photon energy, similarly to photoionisation in atoms. ${ }^{4}$ The absorption cross-section for this process has been calculated ${ }^{5}$ to be several orders of magnitude larger than in bulk. It can affect the performance of a QD laser both through the increased optical loss and through the gain reduction resulting from the removal of carriers from the lasing state. Despite its potential significance, to the best of our knowledge, no experimental evidence of this process and of its effect on laser characteristics has been provided. In this paper, we investigate the emission of an InAs/GaAs $1.3 \mu \mathrm{m}$ QD laser under injection of sub-band gap $1.5 \mu \mathrm{m}, 100 \mathrm{fs}$ pulses, and we find clear evidence of carrier photoexcitation in the strong depletion of laser output. We further confirm this interpretation by directly measuring the pump transmission and induced photocurrent as a function of carrier population in the QDs. The experimental dynamics is well reproduced by

\footnotetext{
* Corresponding author. E-mail: pablo.moreno@epfl.ch.

$\dagger$ Present address: COBRA Research Institute, Eindhoven University of Technology, P.O. Box 513, NL-5600MB Eindhoven, The Netherlands.
}

a rate equation model, which takes into account the experimental photoexcitation cross-section. This process is then shown to play a role in the static and dynamic characteristics of QD lasers.

The QD lasers analyzed were grown by molecular beam epitaxy in the Stranski-Krastanov mode, which has been proved to be a reliable method to provide semiconductor embedded dots. The active layer consists of five layers of self-assembled InAs QDs, covered by $5 \mathrm{~nm}$ thick InGaAs capping layers and separated from each other by a $40 \mathrm{~nm}$ GaAs active layer. The areal dot density of our lens-shaped QDs is $3 \times 10^{10} \mathrm{~cm}^{-2}$. The waveguide was clad by $1.5 \mu \mathrm{m}$ of AlGaAs, n-doped on the substrate side and p-doped on the topside. The ridge was $5 \mu \mathrm{m}$ wide and the cavity is 1.9 $\mathrm{mm}$ long. Both facets were cleaved, providing a $30 \%$ reflectivity. The experimental setup for temporally assessing the dynamics of the lasers is similar to the one described in the work of Hessler et al. ${ }^{6}$ Pump pulses (1.5 $\left.\mu \mathrm{m}, 100 \mathrm{fs}\right)$ from an optical parametric oscillator were injected into the quantum dot laser with a microscope objective. The $1.3 \mu \mathrm{m}$ laser light emitted by the quantum dot laser was timeresolved through up-conversion using a BBO crystal and a gating $810 \mathrm{~nm}$ pulse coming from the Ti:sapphire pump laser. The injection was optimized by maximizing the photocurrent generated between the laser contacts. The maximum average pump power before injection was $250 \mathrm{~mW}$. We note that perturbation by ultrafast pulses has been used to characterize the laser dynamics in bulk, ${ }^{7} \mathrm{QW},{ }^{8}$ and $\mathrm{QD}$ lasers ${ }^{9}$ but only with pump photon energies above the gap. The use of subband gap frequencies here allows us to unambiguously probe the intraband absorption processes.

In Figure 1, the temporal dynamics of the lasing beam after the perturbation at $t=0$ is shown. The different graphs 

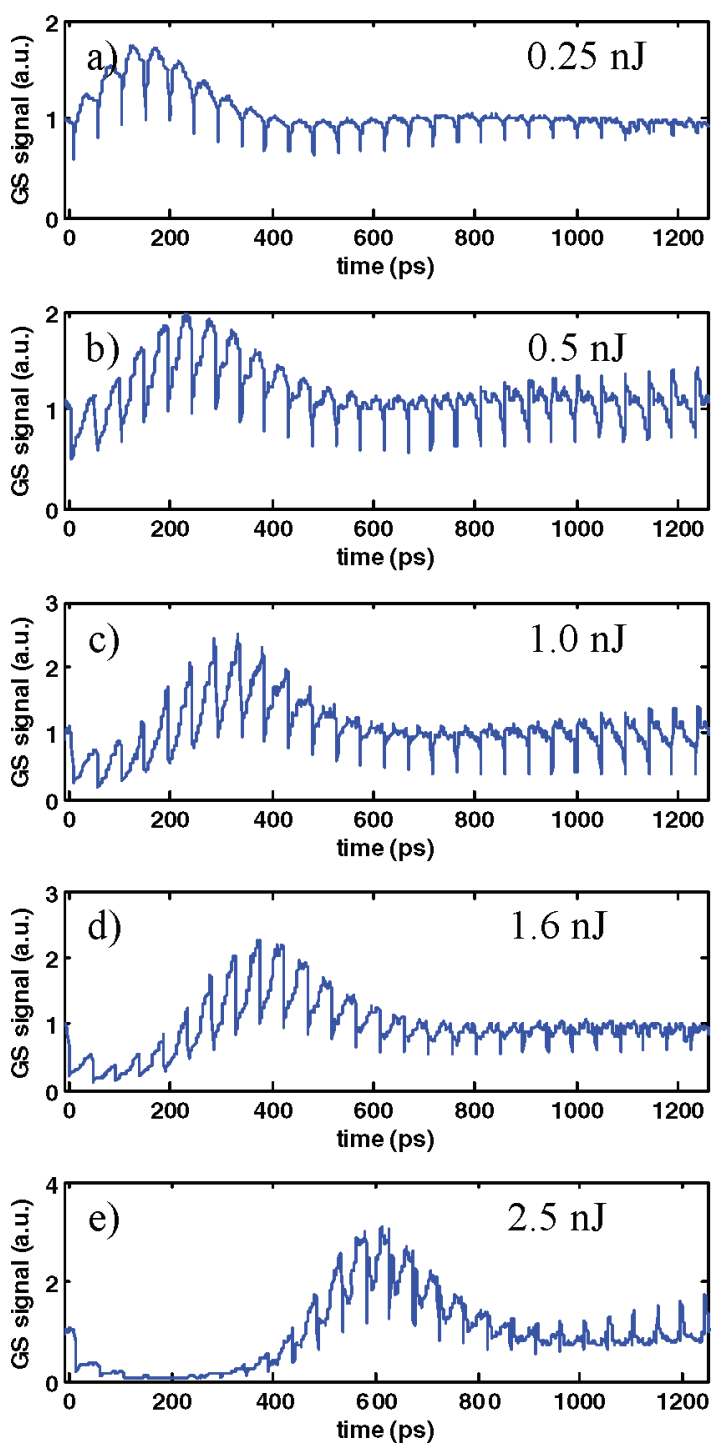

Figure 1. Temporal resolved emission of the laser when it is biased at $4 \mathrm{~V}, 90 \mathrm{~mA}$ for different pump pulse energies. A sudden depletion of the laser emission is followed by highly damped relaxation oscillations. The inset of (e) shows a diagram of the photoexcitation process.

correspond to different energies of the injected pulse. The laser temporal response shows periodic drops of intensity with ultrafast $(0.1-10 \mathrm{ps})$ recovery, superposed to a slower (200-400 ps) relaxation oscillation dynamics. The periodic intensity minima, with a period corresponding to the roundtrip cavity time, correspond to dark pulses. ${ }^{7}, 10$ Indeed, the sudden decrease in laser intensity produced by the pump pulse (through the mechanisms discussed below) propagates back and forth in the cavity even after the re-establishment of carrier population due to the gain $=$ loss condition. At low input powers (see Figure 1a), the dark pulses present only a subpicosecond ultrafast relaxation, which we attribute to crossed two-photon absorption involving one photon at $1.3 \mu \mathrm{m}$ and one photon at $1.5 \mu \mathrm{m}$, as commonly observed. ${ }^{7}$ At higher input powers, Figure $1 \mathrm{~b}-\mathrm{e}$, we notice an additional intensity drop with a slower recovery time (few picoseconds). This dynamics is a strong evidence of gain depletion due to photoexcitation of carriers from the QD ground state and its

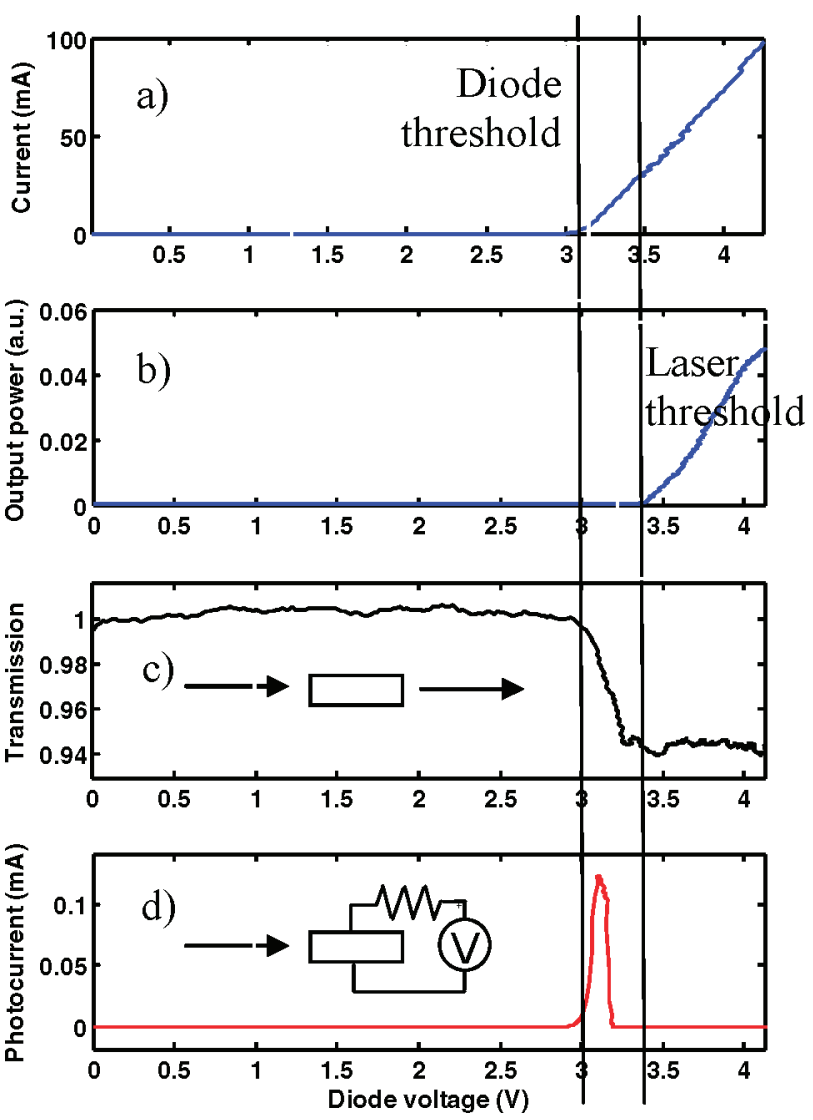

Figure 2. (a) Current as a function of the applied diode voltage. The diode threshold occurs at 3.1 V. (b) Output power of the diode laser. The laser threshold occurs at $3.4 \mathrm{~V}$, which corresponds to 25 $\mathrm{mA}$. (c) Normalized transmitted pump as a function of the applied bias. The inset shows the setup diagram. (d) Photocurrent generated by the $1.5 \mu \mathrm{m}$ pump as a function of the bias voltage.

subsequent recovery through intraband relaxation from higher-energy states. Indeed, carrier relaxation in QDs was already observed to take place in a few ps time scale, both by direct gain recovery experiments ${ }^{11}$ and from the analysis of lasing spectra. ${ }^{12}$ Additional insight is obtained from the relaxation oscillation dynamics. Indeed, if we consider the envelope of the laser response trace of the dark pulses, we observe an initial depletion followed by highly damped relaxation oscillations of the QD laser emission. In particular, at the maximum injected power, Figure 1e, the QD laser is nearly switched off for few hundred picoseconds. This initial depletion cannot be explained by the effect of the two-photon absorption, as this process increases the number of carriers in the active region and would therefore produce an increase of laser intensity. Instead, the photon depletion is a direct consequence of intraband carrier photoexcitation, which promotes carriers (electrons and/or holes) from the lasing state to the continuum, producing a temporary gain depletion that later recovers through relaxation oscillations.

To confirm this interpretation, we have directly measured the effect of QD population on the transmission of the pump beam (Figure 2). In Figure 2a, the $I-V$ curve of the diode laser is shown. This diode presents a relatively large onset voltage of $3.1 \mathrm{~V}$ due to nonannealed contacts. Further increasing the voltage leads to population inversion and 

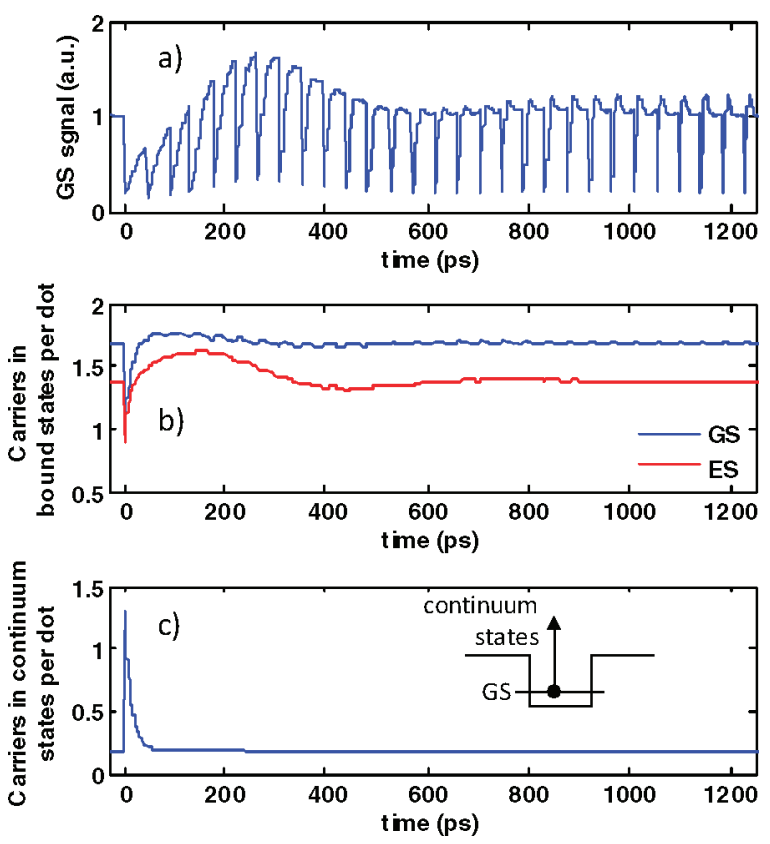

Figure 3. Simulation of the temporal resolved emission of the laser after a pump pulse arrives at $t=0$. Normalized output power (a), normalized population of bound states (b), and of continuum states (c) per dot.

finally lasing at a threshold voltage of $3.4 \mathrm{~V}$, as seen from the light-voltage curve in Figure 2b. At this voltage, the population of the lasing level is clamped. ${ }^{12}$ Under a pulsed injection of $1 \mathrm{~nJ}$, we measured the pump power detected at the output facet, after transmission through the laser. The results, as a function of the voltage, are shown in Figure 2c. The figure reveals important information about the nature of the pump losses. First, the pump losses remain constant up to the diode threshold. Then they increase up to the laser threshold and stay almost constant at higher voltages. This indicates that pump losses are directly associated to carrier photoexcitation from the QD bound states, whose population increases proportionately to the current above the diode threshold and is approximately constant above laser threshold. We note that crossed two-photon absorption, which would produce a strong loss at the pump frequency above laser threshold, has a much smaller effect than photoexcitation in the present experiment. We additionally measured the photocurrent in the QD laser induced by pumping with $1.5 \mu \mathrm{m}$ pulses as a function of the input voltage (Figure $2 \mathrm{~d}$ ). The photocurrent is measured by chopping the pump beam and synchronous lock-in detection of the voltage across a resistance in series to the QD laser. We distinguish three well-defined regions. Below the diode threshold, no carriers are present in the dots and the photocurrent is low. This indicates that the direct two-photon absorption of the pump pulses is negligible. Above the diode threshold, carriers populate dot levels. The absorption of pump pulses (Figure 2c) promotes these carriers to continuum states where they can contribute to conduction, producing a photocurrent. At larger forward bias, the photocurrent is quenched due to the combined effect of reduced internal field and of potential barriers at the heterointerfaces. ${ }^{13}$ This set of measurements

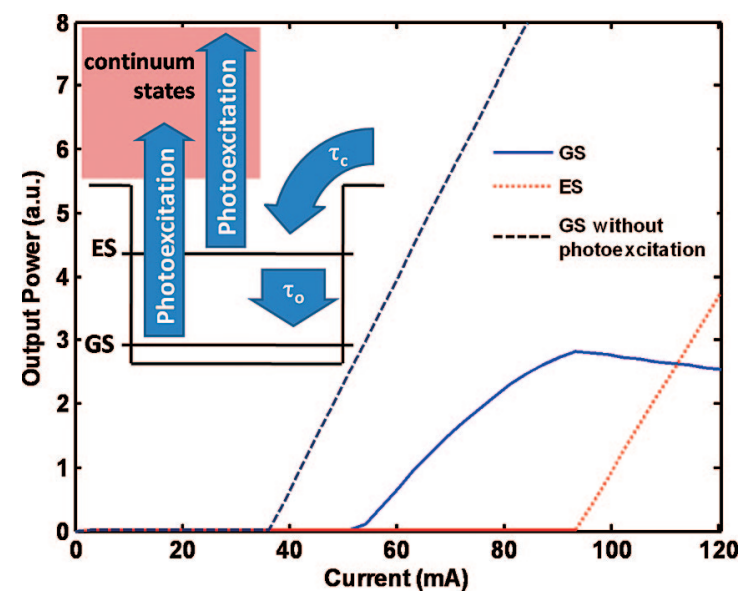

Figure 4. Simulation of the output power as a function of input current for a QD laser with carrier photoexcitation present. As a direct consequence of the photoexcitation mechanism, a reduction of the output power appears when the excited state starts to lase.

fully supports our understanding of intraband photoexcitation as the physical process responsible for the gain depletion observed in Figure 1. In particular, we note that, differently from the case of Platonov et al. ${ }^{9}$ free-carrier loss, related to carriers produced by two-photon absorption of the pump, cannot account for the dynamics observed in our experiments as such free carriers are only observed (Figure 2d) in the presence of QD population, so they are produced by carrier photoexcitation.

We get a detailed insight of the intraband mechanism by modeling the QD laser with a traveling wave rate equation model. This model has successfully explained two-state lasing and carrier dynamics of QD lasers ${ }^{12,14}$ and the characteristics of QD superluminescent diodes. ${ }^{15} \mathrm{We}$ consider the population of three different levels: the ground state (GS), the first excited state (ES) within the dot, and a continuum level corresponding to the wetting layer (WL) and barrier population (see inset of Figure 4 ). $f_{\mathrm{GS}, \mathrm{ES}}$ are the distribution functions of the GS and ES carriers, while $f_{\mathrm{WL}}$ corresponds to the WL population divided by the total number of dots. The rate equations for these variables are:

$$
\begin{gathered}
\frac{\mathrm{d} f_{\mathrm{GS}}}{\mathrm{d} t}=\frac{2 f_{\mathrm{ES}}\left[1-f_{\mathrm{GS}}\right]}{\tau_{0}}-\frac{f_{\mathrm{GS}}\left[1-f_{\mathrm{ES}}\right]}{\tau_{\mathrm{GS}}^{\mathrm{esc}}}-2 B \varphi_{\mathrm{GS}}\left(f_{\mathrm{GS}}-\frac{1}{2}\right)-\frac{f_{\mathrm{GS}}}{\tau_{\mathrm{r}}}- \\
\frac{f_{\mathrm{GS}}}{\tau_{\mathrm{nr}}}-a_{\mathrm{PhE}} f_{\mathrm{GS}}\left(\varphi_{\mathrm{GS}}+\varphi_{\mathrm{ES}}+\varphi_{\mathrm{Pump}}\right) \\
\frac{\mathrm{d} f_{\mathrm{ES}}}{\mathrm{d} t}=\frac{f_{\mathrm{WL}}\left[1-f_{\mathrm{ES}}\right]}{4 \tau_{\mathrm{c}}}-\frac{f_{\mathrm{ES}}\left[1-f_{\mathrm{GS}}\right]}{\tau_{0}}+\frac{f_{\mathrm{GS}}\left[1-f_{\mathrm{ES}}\right]}{2 \tau_{\mathrm{GS}}^{\mathrm{esc}}}-\frac{f_{\mathrm{ES}}}{\tau_{\mathrm{ES}}^{\mathrm{esc}}}- \\
2 B \varphi_{\mathrm{ES}}\left(f_{\mathrm{ES}}-\frac{1}{2}\right)-\frac{f_{\mathrm{ES}}}{\tau_{\mathrm{r}}}-\frac{f_{\mathrm{ES}}}{\tau_{\mathrm{nr}}}-a_{\mathrm{PhE}} f_{\mathrm{ES}}\left(\varphi_{\mathrm{GS}}+\varphi_{\mathrm{ES}}+\varphi_{\mathrm{Pump}}\right) \\
\frac{\mathrm{d} f_{\mathrm{WL}}}{\mathrm{d} t}=G-\frac{f_{\mathrm{WL}}\left[1-f_{\mathrm{ES}}\right]}{\tau_{\mathrm{c}}}+\frac{4 f_{\mathrm{ES}}}{\tau_{\mathrm{ES}}^{\mathrm{esc}}}-\frac{f_{\mathrm{WL}}}{\tau_{\mathrm{r}}}-\frac{f_{\mathrm{WL}}}{\tau_{\mathrm{nr}}}+a_{\mathrm{PhE}}\left(2 f_{\mathrm{GS}}+\right. \\
\left.4 f_{\mathrm{ES}}\right)\left(\varphi_{\mathrm{GS}}+\varphi_{\mathrm{ES}}+\varphi_{\mathrm{Pump}}\right)
\end{gathered}
$$

where $\tau_{0}$ is the intraband relaxation time. A value of $\tau_{0}=7$ ps has been obtained from the ratio of GS and ES threshold currents, ${ }^{12}$ also in agreement with other previous experimental results. ${ }^{16} \tau_{\mathrm{c}}$ is the ES capture time, 1 ps. ${ }^{16} \tau_{\mathrm{r}}$ is the radiative lifetime (measured to be $\approx 1 \mathrm{~ns}$ in these QDs), ${ }^{17} \tau_{\mathrm{nr}}$ the 
nonradiative lifetime (arbitrarily fixed at $\tau_{\mathrm{nr}}=1 \mathrm{~ns}$ ) and $G$ is the carrier injection rate. The escape times $\left(\tau^{\mathrm{esc}}\right)$ are derived by assuming a thermal equilibrium in the absence of external excitation. The gain coefficient $B$ has been fixed to get a saturated gain of $12.5 \mathrm{~cm}^{-1}$. The carrier photoexcitation process is modeled through the last term of the equations, where $a_{\mathrm{PhE}}$ is the rate of carrier photoexcitation in $\mathrm{s}^{-1}$, related to the carrier photoexcitation $(\sigma)$ cross-section through $a_{\mathrm{PhE}}$ $=v_{\mathrm{g}} \sigma \rho$, where $\rho$ is the dot density per unit volume. Notice that here the photon numbers $(\varphi)$ are normalized to the dot number. The total photon number $\varphi$ is the sum of the photon numbers traveling forward $\left(\varphi^{+}\right)$and backward $\left(\varphi^{-}\right)$through the cavity at the GS and the ES energies, which are calculated from:

$$
\begin{aligned}
\frac{\partial \varphi_{\mathrm{GS}, \mathrm{ES}}^{ \pm}}{\partial t} \pm v_{\mathrm{g}} \frac{\partial \varphi_{\mathrm{GS}, \mathrm{ES}}^{ \pm}}{\partial x}= & 2 \delta_{\mathrm{GS}, \mathrm{ES}} B \varphi_{\mathrm{GS}, \mathrm{ES}}^{ \pm}\left(f_{\mathrm{GS}, \mathrm{ES}}-\frac{1}{2}\right)-\frac{\varphi_{\mathrm{GS}, \mathrm{ES}}^{ \pm}}{\tau_{\varphi}}+ \\
& \delta_{\mathrm{GS}, \mathrm{ES}} \frac{\beta}{\tau_{\mathrm{r}}} f_{\mathrm{GS}, \mathrm{ES}}-a_{\mathrm{PhE}}\left(2 f_{\mathrm{GS}}+4 f_{\mathrm{ES}}\right) \varphi_{\mathrm{GS}, \mathrm{ES}}^{ \pm}
\end{aligned}
$$

where $v_{\mathrm{g}}$ is the group velocity, $\beta$ is the spontaneous emission coupling factor, which we have assumed to be $10^{-4}, \tau_{\varphi}$ is the photon lifetime, $17 \mathrm{ps}$, and $\delta_{\mathrm{GS}, \mathrm{ES}}$ is the degeneracy of the GS and ES levels, 2 and 4, respectively.

The results of the model are shown in Figure 3. In Figure $3 \mathrm{a}$, the photon number is plotted as a function of time for conditions similar to the experimental results of Figure 2c. When the pump arrives, at $t=0$, carriers in the bound states are promoted to the continuum states. We represent the temporal evolution of bound states and continuum states in parts $b$ and $c$ of Figure 3, respectively. After the perturbation, carriers relax to the bound states. This capture time affects the risetime of the dark pulse. However, the recovery of the photon density inside the cavity is much slower. When the cavity photons are completely depleted, this recovery is seeded by spontaneous emission, leading to a recovery time as long as $1 \mathrm{~ns}$.

The best fit is obtained with a cross-section for the boundto-continuum states transitions of $6 \times 10^{-17} \mathrm{~cm}^{2}$ for the 1.5 $\mu \mathrm{m}$ incident wavelength. A very similar value of $8 \times 10^{-17}$ $\mathrm{cm}^{2}$ is obtained directly from the pump transmission measurement in Figure 2c, assuming that the pump losses are caused by this effect. Although our experiments do not allow measuring independently the electron and hole crosssections, the value for electrons is expected to be much larger ${ }^{5}$ due to the smaller effective masses. We therefore assume that electron photoexcitation is responsible for all the observed dynamics. The measured cross-section is considerably higher than the corresponding value for bulk materials, ${ }^{18} 6 \times 10^{-18} \mathrm{~cm}^{2}$ because, in quantum dots, there is no need to involve a third particle to satisfy the momentum conservation. Our experimental results show good agreement with previously calculated values, ${ }^{5}$ which suggested a sizedependent intraband cross-section in the range of $10^{-16} \mathrm{~cm}^{2}$. We note that, while we did not investigate the spectral variation of the cross-section, a relatively weak frequency dependence is expected ${ }^{5}$ because photoexcitation involves transitions to a continuum of states in the bands.
The effect of carrier photoexcitation on laser characteristics is twofold, affecting carriers and photons in the cavity. On one hand, it adds free-carrier optical losses, as cavity photons are absorbed in the photoexcitation process. Using the experimentally determined value of the carrier photoexcitation cross-section, $\sigma=8 \times 10^{-17} \mathrm{~cm}^{2}$, we get losses of 0.3 $\mathrm{cm}^{-1}$ when the bound states are completely full. As it has been observed, ${ }^{5}$ this additional loss is often negligible in typical laser cavities. On the other hand, and more importantly, carrier photoexcitation produces a photon-dependent carrier depletion from the lasing state, whose consequences are dramatically evident in the experiments shown in Figure 1 but also relevant in normal laser operation. In fact, the fact that gain decreases (at constant total population) for increasing photon number is a form of gain compression, which affects both the static and the dynamic characteristics. The static differential efficiency is reduced, as more carriers must be injected to maintain a constant gain at increasing photon density. Additionally, the damping of the modulation response is affected by the increased gain compression, thus reducing the maximum modulation frequency. ${ }^{19}$ Both these effects, in principle present in any diode laser, are particularly relevant in QD lasers due on one hand to the larger photoexcitation cross-section and on the other hand to the larger saturation of the lasing state due to the limited density of states, which slows down the intraband relaxation process and consequently the laser dynamics. ${ }^{20}$

As an illustration of these effects, we have calculated the static characteristics of a two-state laser ${ }^{12}$ with and without the presence of carrier photoexcitation (Figure 4). We assumed that the cross-section for $1.5 \mu \mathrm{m}$ photons in our experiments is also valid for $1.3 \mu \mathrm{m}$ cavity photons. We consider the case of a laser (5 QD layers, maximum gain 12.5 and $9 \mathrm{~cm}^{-1}$ total optical losses), where lasing is obtained close to saturation of GS gain, as the effect of photoexcitation will be particularly evident in this situation. We observe multiple consequences of carrier photoexcitation. First, we notice an increase in threshold current (due to the increased optical loss) and a reduction in differential efficiency, as noted above. Additionally, when including photoexcitation, simultaneous lasing on ES and GS, due to the limited intraband relaxation rate, ${ }^{12}$ is more easily observed, as shown in Figure 4. This is related to the additional loss of GS carriers due to photoexcitation, implying a faster increase of ES population. Finally, the GS emission is seen (Figure 4) to decrease above ES threshold, as observed experimentally, ${ }^{21,22}$ while the model not including photoexcitation was not able to predict this decrease. ${ }^{12}$ Indeed, photoexcitation of GS carriers due to ES photons plays a major role in this situation, as the rate of photoexcitation from the GS cannot be compensated by an increase of the clamped ES population. The same effect can account for the surprising observation of negative differential gain observed in the dynamics of twostate lasers. ${ }^{23}$ We note that, in QD lasers operated farther away from gain saturation, the effect of photoexcitation, although relevant, will be less dramatic.

In conclusion, we have reported the first experimental evidence of the photoexcitation process in semiconductor 
quantum dots, whereby carriers are optically excited from bound QD states to continuum states in the barrier. This effect is unambiguously evidenced by the appearance of a depletion in the temporal dynamics of a QD laser after the injection of a sub-band gap pump and further confirmed by transmission and photocurrent measurements.

By including photoexcitation in a laser rate equation model, we were able to fully reproduce the observed dynamics. The photoexcitation cross-section values derived independently from fitting the temporal dynamics and from the direct measurement of pump loss are in very good agreement with each other and with previous theoretical calculations. The cross-section is orders of magnitude larger than in bulk and has a major impact on static and dynamic laser characteristics. Photoexcitation thus plays an important role in QD lasers and should be taken into account in the modeling and design of these devices. While the laser system has been chosen here as a sensitive probe of this process, we note that photoexcitation will play an important role in all QD-based photonic devices, including lasers, amplifiers, light-emitting diodes, photodetectors, and modulators.

Acknowledgment. P. Moreno thanks I. Esquivias for useful discussion. We acknowledge financial support to EUFP6 project "ZODIAC" (contract no. 17140) and to the Swiss National Science Foundation.

\section{References}

(1) Phillips, J.; Bhattacharya, P.; Kennerly, S. W.; Beekman, D. W.; Dutta, M. IEEE J. Quantum Electron. 1999, 35 (6), 936-943.

(2) Sauvage, S.; Boucaud, P.; Brunhes, T.; Immer, V.; Finkman, E.; Gerard, J. M. Appl. Phys. Lett. 2001, 78 (16), 2327-2329.

(3) Sauvage, S.; Boucaud, P.; Gerard, J. M.; Thierry-Mieg, V. Phys. Rev. $B$ 1998, 58 (16), 10562-10567.

(4) Brandsen, B. H., Joachain, C. J., Physics of Atoms and Molecules; Longman: London, 1997.

(5) Asryan, L. V.; Suris, R. A. Semiconductors 2001, 35 (8), 979-979.
(6) Hessler, T.; Haacke, S.; Pleumeekers, J. L.; Selbmann, P. E.; Dupertuis, M. A.; Deveaud, B.; Taylor, R. A.; Doussiere, P.; Bachmann, M.; Ducellier, T.; Emery, J. Y. J. Opt. B: Quantum Semiclassical Opt. 1997, 9 (5), 675-679.

(7) Selbmann, P. E.; Hessler, T. P.; Pleumeekers, J. L.; Dupertuis, M. A.; Deveaud, B.; Dagens, B.; Emery, J. Y. Appl. Phys. Lett. 1999, 75 (24), 3760-3762.

(8) Hense, S. G.; Wegener, M. Phys. Rev. B 1997, 55 (15), 9255-9258.

(9) Platonov, A. V.; Lingk, C.; Feldmann, J.; Arzberger, M.; Bohm, G.; Amann, M. C.; Abstreiter, G. Appl. Phys. Lett. 2002, 81 (7), 11771179.

(10) Zimmermann, J.; Cundiff, S. T.; von Plessen, G.; Feldmann, J.; Arzberger, M.; Bohm, G.; Amann, M. C.; Abstreiter, G. Appl. Phys. Lett. 2001, 79 (1), 18-20.

(11) Borri, P.; Schneider, S.; Langbein, W.; Woggon, U.; Zhukov, A. E.; Ustinov, V. M.; Ledentsov, N. N.; Alferov, Zh. I.; Ouyang, D.; Bimberg, D. Appl. Phys. Lett. 2001, 79 (16), 2633-2635.

(12) Markus, A.; Chen, J.; Paranthoen, C.; Fiore, A.; Platz, C.; GauthierLafaye, O. Appl. Phys. Lett. 2003, xxx1818-1820.

(13) Capasso, F.; Cho, A. Y.; Mohammed, K.; Foy, P. W. Appl. Phys. Lett. 1985, 46 (7), 664-666.

(14) Markus, A.; Rossetti, M.; Calligari, V.; Chek-Al-Kar, D.; Chen, J. X.; Fiore, A.; Scollo, R. J. Appl. Phys. 2006, 100 (11), 113104.

(15) Rossetti, M.; Markus, A.; Fiore, A.; Occhi, L.; Velez, C. IEEE Photonics Technol. Lett. 2005, 17 (3), 540-542.

(16) Sun, K.; Chen, J.; Lee, C.; Kechiantz, A. Nanotechnology 2005, 15301535.

(17) Fiore, A.; Borri, P.; Langbein, W.; Hvam, J. M.; Oesterle, U.; Houdré, R.; Stanley, R. P.; Ilegems, M. Appl. Phys. Lett. 2000, 76 (23), 3430.

(18) Valley, G. C.; Smirl, A. L. IEEE J. Quantum Electron. 1988, 24 (2), 304-310.

(19) Coldren, L. A.; Corzine, S. W. Diode Lasers and Photonic Integrated Circuits; Wiley: New York, 1995.

(20) Fiore, A.; Markus, A. IEEE J. Quantum Electron. 2007, 43 (3-4), 287-294.

(21) Sugawara, M.; Hatori, N.; Ebe, H.; Ishida, M.; Arakawa, Y.; Akiyama, T.; Otsubo, K.; Nakata, Y. J. Appl. Phys. 2005, 97 (4), 053107.

(22) Viktorov, E. A.; Mandel, P.; Tanguy, Y.; Houlihan, J.; Huyet, G. Appl. Phys. Lett. 2005, 87 (5), 053113.

(23) Dagens, B.; Markus, A.; Chen, J. X.; Provost, J.-G.; Make, D.; Le Gouezigou, O.; Landreau, J.; Fiore, A.; Thedrez, B. Electron. Lett. 2005, 41 (6), 323-324.

NL073115A 